\title{
Survey on Erythema Migrans, and Basal Cell Carcinoma in Computer-Aided Diagnosis
}

\author{
Radhakrishnan K R ${ }^{\mathrm{a}, 1}$, Sudalaimuthu $\mathrm{T}^{\mathrm{b}}$ and Dhanalakshmi $\mathrm{R}^{\mathrm{c}}$ \\ ${ }^{a}$ Research Scholar, Department of Computer Science, Hindustan Institute of \\ Technology\& Science, Chennai \\ ${ }^{\mathrm{b}}$ Associate Professor, Department of Computer Science, Hindustan Institute of \\ Technology \& Science, Chennai \\ c Professor, Department of Computer Science, KCG College of Technology, Chennai
}

\begin{abstract}
Lyme disease appears by various means one of the causes is infection from a bite of a black-legged tick which leads to the formation of a rash called Erythema Migrans (EM). This Lyme disease is the direct root of skin cancer and the primary phase is known as Basal Cell Carcinoma (BCC). Skin cancer causes pathological situations it integument the body's surfaces, including skin, hair, nails, and associated glands. Identifying EM and BCC in biomedical representation is a common platform known as biopsies and many works are done in the traditional methodology. But early detection of EM and BCC in the field of medical imaging using computer-aided diagnosis provides more accuracy and rapidity. This survey is classified under two categories; the first is EM detection and the second is the segmentation of BCC. The circumstances of this review are under the base of different algorithms, various methods used by the non-medicals appliance are discussed and compared by the several measures taken by increasing the parameters to improve the accuracy levels.
\end{abstract}

Keywords. Lyme disease, Erythema Migrans, Basal Cell Carcinoma.

\section{Introduction}

Medical image processing [1] is a vast field, researching in this area is a challenging task. Providing accuracy and time consumption are the keystone in image processing. Diseases identification, detection [2,3], classification, and clustering in this field are still not optimal. Various diseases (such as Tumors, Cancer, Skin, Retina, etc.) are still under evolution, and identifying them by computer-aided diagnosis is not ample. According to various reviews and studies on natural human calamities, the World Health Organization (WHO) gives an alert that skin disease is one of the most communal and stimulating sources of human illness. Very commonly, [4] it is known that the cause of cancer is not known for many cases but once it is identified at the beginning it can be cured. It is

\footnotetext{
${ }^{1}$ Radhakrishnan KR, Department of CS, Hindustan Institute of Technology\&Science, chennai, India.

E-mail: krishna.kayaar@gmail.com.
} 
vulnerable to people and it appears through blood, bones, lungs, skin, and many other means. From numerous research and studies [5-7], it is come to know that the cause of cancer through the skin occurs primarily on areas of sun-exposed skin, involves all layers of the skin, and through many mediums (like the scalp, face, lips, ears, neck, chest, arms and hands, and on the legs too) and has several etiology (such as infections, tumors, or inflammation UV radiations, exposure of sunlight). Skin cancer [7,8] through infections also ensues by insect bites. From the regions of northern Midwest and eastern coasts of the United States as well as in southeastern Canada insects so-called black-legged tick or deer tick bites trigger the bacteria Borrelia Mayonii and so engenders Lyme disease. The very foremost stage of Lyme disease [9] is a rash appearance recognized as [10,11] Erythema Migrans (EM). Approximately 70 to 80 percent of people with Lyme disease have this kind of rash. Very often the rash looks like a bulls-eye and for some individual guises like a solid circle. And it is stated by doctors and many research specialists that Lyme disease is the direct cause of skin cancer. The very preliminary step of Lyme disease is [12-14] Basal cell carcinoma or Basal cell cancer (BCC) is an early category of skin cancer. This nature of cancer transpires by the formation of new cells inside the skin as old ones perish off. BCC often appears as a fairly transparent bump on the skin, though it can take other forms. Aspects that are stated and executed as previous work through various image acquisition techniques so-called noise reduction, implementation of various segmentation algorithms, feature extractions process (like Color Features: Min, Max, Mean, SD, and Entropy; and Textures Features: contrast, correlation, and energy), and classification of diseases have resulted in many downsides $[15,16]$.

\section{Surveys}

The review work in this field is categorized into two: detection of Erythema Migrans and feature extraction, and segmentation methods of Basal Cell Carcinoma to detect skin cancer.

\subsection{Erythema Migrans}

Based on the work of Philippe M. Burlina et al. [17], from various experimental analyses, it is proved that machine-based testing outcome better results than manual testing called biopsies. The work was implemented by the operations mean and rescale applied to the raw image to obtain pre-processed image through Image Net [18] and with various Deep Learning Techniques [12,19-23]. Data augmentation functions flip, blur, color contrast, saturate, sharpen, and color balance are performed. [24] ResNet 50 a Deep Convolutional Neural Network model was implemented in the platform Keras and Tensor flow. In the training model, Stochastic Gradient Descent was used to improve the training speed and accuracy. The categorical cross-entropy loss function also named softmax loss function was used to train the Convolutional Neural Network model ResNet 50 to produce the probability-based classes. But entropy loss function stopped at every 10 epochs. So, the dynamic learning rate schedule was given as a constant. This training was held for four classes EM (Erythema Migrans), Normal, Tinea Corporis(TC), and Herpes Zoster(HZ). And later it was reduced to two class classifiers EM and Non-EM classes (Normal, TC, HZ). [25, 26] The training model of four class and two class classifiers was measured 
using the metrics accuracy, F1, sensitivity, specificity, positive predictive value, negative predictive value, kappa score, ROC. Implementation, training, and metric measurement were done with the data set of 1834 images (initially it was 6000 images before preprocessing).

Pegah Kharazmi et al. [27] detected and segment vascular structure from dermoscopy images (dataset of 759 images from three different sources). [28] Independent Component Analysis (ICA) method was applied to decompose the [29] dermoscopic images. Due to this edge detection was conceivable from input images termed image decomposition. The skin layer components melanin and hemoglobin are retrieved [30]. This hemoglobin component extracted achieved a better performance to get RGB values calculated through the Mahalanobis distance. The values of RGB assisted to perform Kmeans cluster to segregate three different classes [28] termed as pigmented, normal, and erythema migrans.

This study from Ramy Abdlaty et al. [31] states the possible measures of Erythema was assessed from Hyperspectral Images (HSI) [13]. The background segregation of the hyperspectral images shaped from the preprocessed image and the segregation of and the white standards from the preprocessed image lead to spectral reflectance images. Image registration was boosted by two inputs digital images (RGB) and the output from spectral reflectance image. [32,33] The Visual Assessment (VA) declares the erythema regions through the X-OR correlation to produce the corrected images. [34] The performance evaluation was analyzed by the contradiction of colored digitalized images Red-GreenBlue (RGB) pigments. The [6] Hyper Spectral Images (HSI) and the RGB pigments are analyzed and classified via the smoothing of images by a low pass filter called SavitzkyGolay and the results are compared with colored images. From the analysis, two major work phase was stated; firstly it was known that to remove low informative bands column subset selection was framed based on the matrix rank [35] representation by Frobenius. In the second phase, the Weiner filter was applied for the noise removal from the outputs spectral reflectance images, registered images, and corrected images.

Examined by Philippe M. Burlina et al. [36] on skin lesions and detecting Erythema Migrans (EM) $[9,11,37]$ using Artificial Intelligence (AI) and Deep Learning (DL) methods $[38,39]$ is the main approach of this study. Early accurate identification of EM avoids rheumatology, neurology, and complications in cardiac. Comparison of the clinical-based skin conditions with Erythema Migrans detection using Artificial Intelligence models and Deep Learning methods was implemented and tested. The clinical skin conditions are tinea corporis, cellulitis, erythema multiforme, herpes zoster, and non-pathogenic normal skin. Complications of multi-class classification with high complexity along with incorporations of clinical-based binary classifications are taken into consideration [40]. The models and methods of AI and DL were trained and tested with images available publicly and also tested with images obtained from clinical data. These trained models and implemented methods were measured by ROC and with the gold standard. According to the metrics [4], public images in DL models produced an accuracy level of $71.58 \%$ to $94.23 \%$ for classification of 8- class problem [41] (having EM and other skin pathology) with binary classification (of EM and non - pathological skin). From the study, it was defined that the [42] DL system helps in prescreening for EM diagnosis as per the dataset [43]. 


\subsection{BCC Segmentation and Feature Extraction}

By Wangting Zhou et al. [44] Malignant Melanoma (MM) and Basal Cell Carcinoma (BCC) [45] tumor growth detection were measured by the functional and structural variations and the study of biopsies in dermatologic condition was the mean in this paper. The apparent skin features and the required parameter data were lacking from the conventional optic imaging techniques to describe the skin disease pathophysiology correlations. Due to the issue [46,47], All Optically Integrated Photoacoustic / Optical Coherence Tomography (AOIP / OCT) preclinical device was suggested. This AOIP / OCT device provided a free label of certain features. Table 1.1 describes the features list for the growth of the tumor by pathophysiologic correlations in MM [48, 49].

Table 1. Feature extraction and classifiers used in BCC classification.

\begin{tabular}{|l|l|l|}
\hline Methods & Features & Classifiers \\
\hline AOPA / OCT [44] & $\begin{array}{l}\text { Vascular, Blood flow velocity, Heterogene- } \\
\text { ity of blood flow, Tissue microstructure } \\
\text { changes, Pigment structures, Cytologic fea- } \\
\text { tures. }\end{array}$ & - \\
\hline Auto Encoder [50] & Patient profile, SAE feature leaning & SoftMax \\
\hline Otsu's Method [27] & Vascular Features & $\begin{array}{l}\text { Simple Logistics, Naïve Bayes, } \\
\text { MLP, Random Forest }\end{array}$ \\
\hline
\end{tabular}

[51] By the measurable metrics of this device, the functional and morphological parameters are rehabilitated due to the impact of spatial-temporal heterogeneity of MM and BCC. Distinguishing the validation criteria of vivo [52,53] from the imaging biomarkers and ex vivo from MM was also provided by the correlation analysis. The device was measured by the ROC analysis had AOIP / OCT parameters improved with the accuracy of MM with $68.4 \%$ and $\mathrm{BCC}$ with $95.8 \%$ correspondingly. As a result, this paper indicates that the accurate diagnosis of clinically translatable technologies was possible.

Early detection by Komal Sharma et al. [54] and segmentation of the BCC by Ramandeep Kaur et al. [55] to detect skin cancer was suggested in this work and also analyzed with [8] previous methods with the flow of preprocessing, feature extraction [1], classification, and measures. [56,57] The input image was preprocessed and smoothly segregated the foreground and the background of the image by the k-means cluster along with the Particle Swarm Optimization (PSO) to improve the quality of background separation of the image. Speed Up Robust Features (SURF) and Scale Invariant Feature Tra nsform (SIFT) were implemented for feature extraction. Artificial Neural Network was trained to get better performance with the classification strategies and also measured with the metrics precision, accuracy, True Positive Ratio (TPR), and False Positive Ratio (FPR).

The work by Kharazmi P et al. [50] is classified into two; To learn the framework of unsupervised features using the [58] Sparse Encoder (SE), and to study the vascular features directly from the given image with its hidden characteristics. [59] Feature maps are derived from the filters that are considered by the individual weights of the learned kernel. Table 1 illustrates the features extracted from the sparse encoder. These feature maps helped to diminish the dimensions and to relate with the records of the patients. The outcome of the feature maps was fed to the SoftMax classifier for classifying the BCC. 
From the author, Luca Fania et al. [60] it was explained that due to exposure to sun and age population skin cancer has become a common occurrence worldwide. Nonmelanoma skin cancer has a subtype [7, 61, 62] Basal Cell Carcinoma (BCC) is a very common type worldwide and started to spread globally. [63] People with an illness caused by this tumor die low in number. Individuals who cross to the next stage without perceiving are more in numbers. A survey in this field started with the pathophysiology till the novel approach therapeutic was taken. The diagnosis for BCC improved with different prognostic values along with the improvement in device strategies like reflectance confocal microscope, and recent dermoscopy images [19,64,65] and clinical features $[13,14]$. Even though the initial stage of treatment was surgical to BCC many local non-surgical treatments are available. Depending on the patient's environmental and genetic behavioral collaborations BCC formation is possible. To treat the advanced level $\mathrm{BBC}$ or metastatic $\mathrm{BCC}$ hedgehog signaling like sonidegib, and vismodegib are represented in this pathogenesis.

\section{Result and Discussions}

As per the above survey on Erythema Migrans, Feature extraction, and BCC segmentation its performance analysis is discussed according to the methods applied and the accuracy levels obtained based on the data set used. According to Figure 1 The Hyperspectral

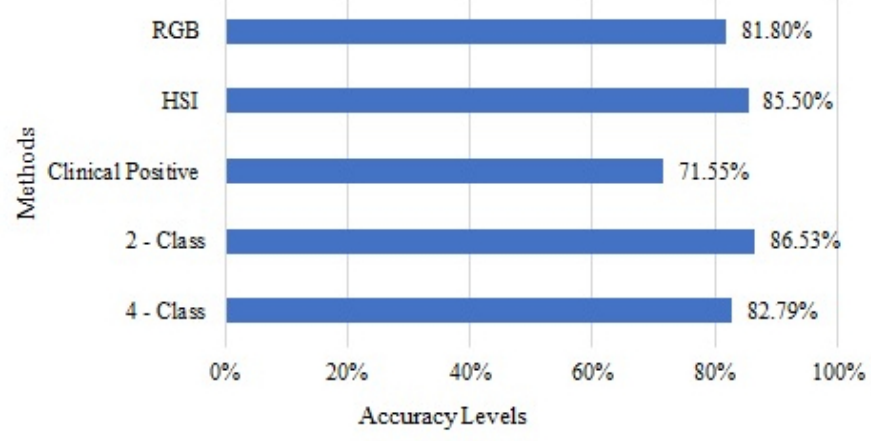

Figure 1. Erythema migrans performance.

Image (HSI) [31], and the Red - Green - Blue (RGB) pigment extraction for the erythema migrans detection resulted in an accuracy level of $85.5 \%$ and $81.8 \%$ respectively. The class-based optimization [17] in 2 class, 4 class, and clinical positive classifiers produced the accuracy outcome in the range of $86.5 \%, 82.79 \%$, and $71.55 \%$ respectively. Methods and techniques used to segment Basal Cell Carcinoma for skin cancer have resulted by Artificial Neural Network with an accuracy of 97.9\%, Sparse AutoEncode (SAE) feature extraction obtained an accuracy of $84.7 \%$, HD-OCT performed the four different models as AlexNet, GoogLE Net, VGG-16, and VGG-19 outcome with the accuracies of $91.6 \%$, $74.4 \%, 93.5 \%$, and $89.1 \%$ respectively. The shape and erythema methods produced an accuracy of $79.1 \%$ and $66.7 \%$ which is represented in Figure 2. 


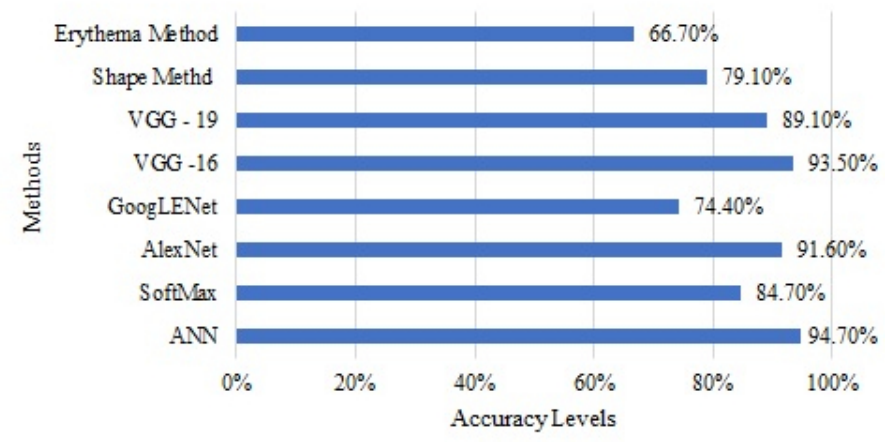

Figure 2. Basal Cell Carcinoma Performance.

\section{Conclusion}

From the consideration of previous work, analyzing various Deep Neural Network for segmentation (Fast RCNN, Faster RCNN, and U-Net ) is considered to be the further study in this area. The keynote in measuring the outcome of medical image processing is accuracy and rapidity. Not enough statistics were done on these circumstances. The vision of this proposal is to analyze the techniques of Deep Learning algorithms to provide the best algorithm using the measurement parameters with various comparison studies. Skin diseases are a massive problem in the world, and there is an alarming necessity to get them into rheostat at the primary stage. so the actual actions can be taken up as soon as possible. Furthermore, the number of dermatologists is quite low as associated with the subjects, which is the scope. So, computer-aided analysis is a boon in such situations as they not only dilute the job of dermatologists but also add effects to their work by reducing the diagnosing time.

\section{References}

[1] Bhuiyan MA, Azad I, Uddin MK. Image processing for skin cancer features extraction. International Journal of Scientific \& Engineering Research. 2013 Feb;4(2):1-6.

[2] Alsarori FA, Kaya H, Rahebi J, Popescu DE, Hemanth DJ. Cancer Cell Detection through Histological Nuclei Images Applying the Hybrid Combination of Artificial Bee Colony and Particle Swarm Optimization Algorithms. International Journal of Computational Intelligence Systems. 2020 Sep;13(1):1507-16.

[3] Ambika M, Raghuraman G, SaiRamesh L, Ayyasamy A. Intelligence-based decision support system for diagnosing the incidence of hypertensive type. Journal of Intelligent \& Fuzzy Systems. 2020 Jan 1;38(2):1811-25.

[4] Burlina PM, Joshi NJ, Ng E, Billings SD, Rebman AW, Aucott JN. Automated detection of erythema migrans and other confounding skin lesions via deep learning. Computers in biology and medicine. 2019 Feb 1;105:151-6.

[5] Ambika M, Raghuraman G, SaiRamesh L. Enhanced decision support system to predict and prevent hypertension using computational intelligence techniques. Soft Computing. 2020 Feb 14:1-12.

[6] Augustus Devarajan A, SudalaiMuthu T. A cloud storage monitoring system using deduplication and file access pattern. International Journal of Recent Technology and Engineering. 2019 Sep;8(3):1972-6.

[7] Gandhi SA, Kampp J. Skin Cancer Epidemiology, Detection, and Management. The Medical Clinics of North America. 2015 Sep 2;99(6):1323-35. 
[8] Masood A, Ali Al-Jumaily A. Computer aided diagnostic support system for skin cancer: a review of techniques and algorithms. International journal of biomedical imaging. 2013 Oct 30;2013:1-22.

[9] Bhate C, Schwartz RA. Lyme disease: Part I. Advances and perspectives. Journal of the American Academy of Dermatology. 2011 Apr 1;64(4):619-36.

[10] Lipsker D, Lieber-Mbomeyo A, Hedelin G. How accurate is a clinical diagnosis of erythema chronicum migrans? Prospective study comparing the diagnostic accuracy of general practitioners and dermatologists in an area where lyme borreliosis is endemic. Archives of dermatology. 2004 May 1;140(5):620-1.

[11] Nadelman RB. Erythema migrans. Infectious Disease Clinics. 2015 Jun 1;29(2):211-39.

[12] Huang H. Automatic translucency detection of basal cell carcinoma (BCC) via deep learning methods (Doctoral dissertation, University of British Columbia).[17.] He Huang, Automatic Translucency Detection of Basal Cell Carcinoma (BCC) Via Deep Learning Methods, A Thesis, The University of British Columbia, 2018.

[13] Kim DP, Kus KJ, Ruiz E. Basal cell carcinoma review. Hematology/Oncology Clinics. 2019 Feb $1 ; 33(1): 13-24$.

[14] Marzuka AG, Book S. Basal cell carcinoma: pathogenesis, epidemiology, clinical features, diagnosis, histopathology, and management. The Yale journal of biology and medicine. 2015 Jun 1;88(2):167-79.

[15] Muthu TS, Kumar KR. Hybrid predictive approach for replica replacement in data grid. In2017 4th International Conference on Advanced Computing and Communication Systems (ICACCS) 2017 Jan 6 (pp. 1-5). IEEE.

[16] Sarah I, Soundarya K, Dhanalakshmi R, Deenadayalan T. DYS-I-CAN: An Aid for the Dyslexic to improve the skills using Mobile Application. In2020 International Conference on System, Computation, Automation and Networking (ICSCAN) 2020 Jul 3 (pp. 1-5). IEEE.

[17] Burlina PM, Joshi NJ, Ng E, Billings SD, Rebman AW, Aucott JN. Automated detection of erythema migrans and other confounding skin lesions via deep learning. Computers in biology and medicine. 2019 Feb 1;105:151-6.

[18] Krizhevsky A, Sutskever I, Hinton GE. Imagenet classification with deep convolutional neural networks. Advances in neural information processing systems. 2012;25:1097-105.

[19] Lallas A, Apalla Z, Argenziano G, Longo C, Moscarella E, Specchio F, Raucci M, Zalaudek I. The dermatoscopic universe of basal cell carcinoma. Dermatology practical \& conceptual. 2014 Jul;4(3):1124.

[20] LeCun Y, Bengio Y, Hinton G. Deep learning. nature. 2015 May;521(7553):436-44.

[21] Ajay H, Rao AR, Balavanan M, Lalit R. A Novel Cardiac Arrest Alerting System using IoT. International Journal of Science Technology \& Engineering. 2017;3(10):78-83.

[22] Anand J, Dhanalakshmi M, and Raja PPJ. Smart Indication System for Spinal Cord Stress Detection. International Journal of Recent Technology and Engineering. 2019 Sep;8(3):6164-8.

[23] Wang H, Liu S, Guo M. 6C model construction and future prospects of innovation ecosystem research based on ecological theory. Arabian Journal of Geosciences. 2021 Jun;14(11):1-5.

[24] He K, Zhang X, Ren S, Sun J. Deep residual learning for image recognition. InProceedings of the IEEE conference on computer vision and pattern recognition 2016 (pp. 770-778).

[25] Kharazmi P, Lui H, Stoecker WV, Lee T. Automatic detection and segmentation of vascular structures in dermoscopy images using a novel vesselness measure based on pixel redness and tubularness. InMedical Imaging 2015: Computer-Aided Diagnosis 2015 Mar 20 (Vol. 9414, p. 94143M). International Society for Optics and Photonics.

[26] Mazori DR, Orme CM, Mir A, Meehan SA, Neimann AL. Vesicular erythema migrans: an atypical and easily misdiagnosed form of Lyme disease. Dermatology online journal. 2015;21(8):1-5.

[27] Kharazmi P, AlJasser MI, Lui H, Wang ZJ, Lee TK. Automated detection and segmentation of vascular structures of skin lesions seen in Dermoscopy, with an application to basal cell carcinoma classification. IEEE journal of biomedical and health informatics. 2016 Dec 8;21(6):1675-84.

[28] Tsumura N, Haneishi H, Miyake Y. Independent-component analysis of skin color image. JOSA A. 1999 Sep 1;16(9):2169-76.

[29] Argenziano G, Soyer HP, De Giorgio V, Piccolo D, Carli P, Delfino M, Ferrari A, Hofmann-Wellenhof R, Massi D, Mazzocchetti G, Scalvenzi M. Interactive atlas of dermoscopy. Milan, Italy: Edra Medical Publishing and New Media, 2000.

[30] Di Leo G, Paolillo A, Sommella P, Fabbrocini G, Rescigno O. A software tool for the diagnosis of melanomas. In2010 IEEE Instrumentation \& Measurement Technology Conference Proceedings 2010 May 3 (pp. 886-891). IEEE. 
[31] Abdlaty R, Doerwald-Munoz L, Madooei A, Sahli S, Yeh SC, Zerubia J, Wong RK, Hayward JE, Farrell TJ, Fang Q. Hyperspectral imaging and classification for grading skin erythema. Frontiers in Physics. 2018 Aug 28;6(72):1-10.

[32] Pramanik T, Khatiwada B, Pandit R. Color vision deficiency among a group of students of health sciences. Nepal Med Coll J. 2012 Dec 1;14(4):334-6.

[33] Trotti A, Colevas AD, Setser A, Rusch V, Jaques D, Budach V, Langer C, Murphy B, Cumberlin R, Coleman CN, Rubin P. CTCAE v3. 0: development of a comprehensive grading system for the adverse effects of cancer treatment. InSeminars in radiation oncology 2003 Jul 1 (Vol. 13, No. 3, pp. 176-181). WB Saunders.

[34] Madooei A, Abdlaty RM, Doerwald-Munoz L, Hayward J, Drew MS, Fang Q, Zerubia J. Hyperspectral image processing for detection and grading of skin erythema. InMedical Imaging 2017: Image Processing 2017 Feb 24 (Vol. 10133, p. 1013322). International Society for Optics and Photonics.

[35] Liu G, Lin Z, Yan S, Sun J, Yu Y, Ma Y. Robust recovery of subspace structures by low-rank representation. IEEE transactions on pattern analysis and machine intelligence. 2012 Apr 10;35(1):171-84.

[36] Burlina PM, Joshi NJ, Mathew PA, Paul W, Rebman AW, Aucott JN. AI-based detection of erythema migrans and disambiguation against other skin lesions. Computers in Biology and Medicine. 2020 Oct $1 ; 125: 103977$.

[37] Steere AC, Sikand VK. The presenting manifestations of Lyme disease and the outcomes of treatment. New England Journal of Medicine. 2003 Jun 12;348(24):2472-4.

[38] Huang G, Liu Z, Van Der Maaten L, Weinberger KQ. Densely connected convolutional networks. InProceedings of the IEEE conference on computer vision and pattern recognition 2017 (pp. 4700-4708).

[39] Szegedy C, Ioffe S, Vanhoucke V, Alemi AA. Inception-v4, inception-resnet and the impact of residual connections on learning. InThirty-first AAAI conference on artificial intelligence 2017 Feb 12 (pp. 4278-4284).

[40] Liu Y, Jain A, Eng C, Way DH, Lee K, Bui P, Kanada K, de Oliveira Marinho G, Gallegos J, Gabriele S, Gupta V. A deep learning system for differential diagnosis of skin diseases. Nature medicine. 2020 Jun;26(6):900-8.

[41] Esteva A, Kuprel B, Novoa RA, Ko J, Swetter SM, Blau HM, Thrun S. Dermatologist-level classification of skin cancer with deep neural networks. Nature. 2017 Feb;542(7639):115-8.

[42] Zoph B, Vasudevan V, Shlens J, Le QV. Learning transferable architectures for scalable image recognition. InProceedings of the IEEE conference on computer vision and pattern recognition 2018 (pp. 8697-8710).

[43] Khan A, Sohail A, Zahoora U, Qureshi AS. A survey of the recent architectures of deep convolutional neural networks. Artificial Intelligence Review. 2020 Dec;53(8):5455-516.

[44] Niranjana S, Hareshaa SK, Irene ZB, Anand J. Smart Monitoring System for Asthma Patients. International Journal of Electronics and Communication Engineering. 2020 May;7(5):5-9.

[45] Stücker M, Esser M, Hoffmann M, Memmel U, Hirschmüller A, Von Bormann C, Hoffmann K, Altmeyer P. High-resolution laser Doppler perfusion imaging aids in differentiating between benign and malignant melanocytic skin tumours. Acta dermato-venereologica. 2002 Jan 1;82(1):25-9.

[46] Du L, Qin H, Ma T, Zhang T, Xing D. In vivo imaging-guided photothermal/photoacoustic synergistic therapy with bioorthogonal metabolic glycoengineering-activated tumor targeting nanoparticles. Acs Nano. 2017 Sep 26;11(9):8930-43.

[47] Zhou W, Chen Z, Yang S, Xing D. Optical biopsy approach to basal cell carcinoma and melanoma based on all-optically integrated photoacoustic and optical coherence tomography. Optics letters. 2017 Jun 1;42(11):2145-8.

[48] Ranjana P, Sridevi SL, Muthu TS, Gnanaraj VV. Machine Learning Algorithm in Two wheelers fuel Prediction. In2019 1st International Conference on Innovations in Information and Communication Technology (ICIICT) 2019 Apr 25 (pp. 1-5). IEEE.

[49] Revathi J, Anitha J, Hemanth DJ. Training feedforward neural network using genetic algorithm to diagnose left ventricular hypertrophy. Telkomnika. 2020 Jun 1;18(3):1285-91.

[50] Kharazmi P, Kalia S, Lui H, Wang ZJ, Lee TK. A feature fusion system for basal cell carcinoma detection through data-driven feature learning and patient profile. Skin research and technology. 2018 May;24(2):256-64.

[51] Zalaudek I, Kreusch J, Giacomel J, Ferrara G, Catricala C, Argenziano G. How to diagnose nonpigmented skin tumors: a review of vascular structures seen with dermoscopy: part I. Melanocytic skin tumors. Journal of the American Academy of Dermatology. 2010 Sep 1;63(3):361-74. 
[52] Longo C, Zalaudek I, Argenziano G, Pellacani G. New directions in dermatopathology: in vivo confocal microscopy in clinical practice. Dermatologic clinics. 2012 Oct 1;30(4):799-814.

[53] Yang Z, Chen J, Yao J, Lin R, Meng J, Liu C, Yang J, Li X, Wang L, Song L. Multi-parametric quantitative microvascular imaging with optical-resolution photoacoustic microscopy in vivo. Optics express. 2014 Jan 27;22(2):1500-11.

[54] Sharma K, Madaan S. Improved Segmentation algorithm using PSO and K-means for Basal Cell Carcinoma Classification from Skin Lesions. International Journal of Innovative Technology and Exploring Engineering (IJITEE). 2019 July;8(954):2278-3075.

[55] Ramandeep Kaur, Gagandeep, Parveen Kumar, Geetanjali Babbar. An Enhanced and Automatic Skin Cancer Detection using K-Mean and PSO Technique. International Journal of Innovative Technology and Exploring Engineering (IJITEE). 2019 July;8(9S):634-9.

[56] Sudalai Muthu T, Rohini A. A correlative scrutiny for improving the career guidance links in social network. International Journal of Engineering and Advanced Technology. 2019 Oct;9(1):1466-70.

[57] SudalaiMuthu T, RameshKumar K, Sarukesi K. A Weight based replica replacement algorithm in datagrid. Journal of Advanced Research in Dynamical and Control Systems. 2017;9(6):1164-78.

[58] Ng A. CS294A lecture notes: sparse autoencoder. 2010.

[59] Krizhevsky A, Hinton G. Learning multiple layers of features from tiny images.Master's thesis, Department of Computer Science, University of Toronto; 2009.

[60] Fania L, Didona D, Morese R, Campana I, Coco V, Di Pietro FR, Ricci F, Pallotta S, Candi E, Abeni D, Dellambra E. Basal cell carcinoma: from pathophysiology to novel therapeutic approaches. Biomedicines. 2020 Nov;8(449):1-38.

[61] McCormack CJ, Kelly JW, Dorevitch AP. Differences in age and body site distribution of the histological subtypes of basal cell carcinoma: a possible indicator of differing causes. Archives of dermatology. 1997 May 1;133(5):593-6.

[62] Scrivener Y, Grosshans E, Cribier B. Variations of basal cell carcinomas according to gender, age, location and histopathological subtype. British Journal of Dermatology. 2002 Jul;147(1):41-7.

[63] Trakatelli M, Morton C, Nagore E, Ulrich C, Del Marmol V, Peris K, Basset-Seguin N. Update of the European guidelines for basal cell carcinoma management. European Journal of Dermatology. 2014 May;24(3):312-29.

[64] Lallas A, Apalla Z, Ioannides D, Argenziano G, Castagnetti F, Moscarella E, Longo C, Palmieri T, Ramundo D, Zalaudek I. Dermoscopy in the diagnosis and management of basal cell carcinoma. Future Oncology. 2015 Nov;11(22):2975-84.

[65] Lallas A, Argenziano G, Zendri E, Moscarella E, Longo C, Grenzi L, Pellacani G, Zalaudek I. Update on non-melanoma skin cancer and the value of dermoscopy in its diagnosis and treatment monitoring. Expert review of anticancer therapy. 2013 May 1;13(5):541-58. 